\title{
AN ARGUMENTATION-BASED APPROACH FOR DIALOG MOVE SELECTION (EXTENDED ABSTRACT)
}

\author{
Leila Amgoud $^{\mathrm{a}} \quad$ Nabil Hameurlain ${ }^{\mathrm{b}}$ \\ ${ }^{a}$ IRIT, Toulouse France \\ amgoud@irit.fr \\ b LIUPPA, University of Pau, France \\ nabil.hameurlain@univ-pau.fr
}

In a multi-agent system, agents need the ability to engage in dialogs such as the ones identified in [2], namely persuasion and negotiation. Work in the literature has focused on defining formal models for these dialog types. Generally, a dialog system contains the following three components: the agents involved in the dialog (i.e their mental states), a dialog protocol and a set of strategies. The dialog protocol is the set of rules of encounter governing the high-level behavior of interacting agents. A protocol defines among other things:

- the set of permissible acts (eg. asking questions, making offers, presenting arguments, etc.);

- the legal replies for each act.

A dialog protocol identifies the different possible replies after a given act. However, the exact act to utter at a given step of the dialog is a strategy matter. While the protocol is a public notion, strategy is crucially an individualistic matter. A strategy can be seen as a two steps decision process:

1. among all the possible replies allowed by the protocol, to choose the move to play. For instance, in a negotiation dialog, the protocol may allow after an offer act the following moves: accepting/rejecting the offer or making a new offer.

2. to choose the content of the move if any. In the above example, if the agent chooses to make a new offer, it may decide among different alternatives the best one to propose.

In most works on modeling dialogs, the definition of a protocol poses no problems. However, the situation is different for dialog strategies. There is no methodology and no formal models for defining them. There is even no consensus on the different ingredients involved when defining a strategy.

This paper argues that the strategy is a decision problem in which an agent tries to choose among different alternatives the best option, which according to its beliefs, will satisfy at least its most important goals. Two different kinds of goals are involved when selecting the next act to play and its content:

Strategic goals: For choosing the type of act to utter, an agent refers to what we call strategic goals. By strategic goals we mean the meta-level goals of the agent such as "minimizing the dialog time", "selling at the end of the dialog", etc. Suppose that at a given step of a negotiation dialog, an agent has to choose between making an offer and asking a question. If the agent wants to minimize the dialog time then it would choose to make an offer instead of spending more time in questions. However, if the agent wants to get a maximum of information about the wishes of the other agent, then the agent would decide to ask a question. These goals are generally independent of the subject of the dialog. If the agents are negotiating the place of a next meeting, then those goals are not related to the place. 
Functional goals: The goals of the agent which are directly related to the subject of the dialog are called functional goals. They represent what an agent wants to achieve or to get regarding the subject of the dialog. Let us take the example of the agent negotiating the place of a meeting. The agent may prefer a place that is not warm and not expensive. These functional goals are involved when selecting the content of a move. In a negotiation, an agent proposes offers that satisfy such goals.

As for goals, the beliefs involved in the two decision problems (selecting an act and a content) are also of different nature:

Strategic beliefs: which are the meta-level beliefs of the agent. They may represent the beliefs of the agent about the dialog and about the other agents involved in the dialog. In negotiation dialogs where agents are trying to find a common agreement, agents may intend to simulate the reasoning of the other agents. Thus it is important for each agent to consider the beliefs that it has on the other agents'goals and beliefs. Indeed, a common agreement can be more easily reached if the agents check that their offers may be consistent with what they believe are the goals of the others.

Basic beliefs: represent the beliefs of the agent about the environment and the subject of the dialog. Let us consider again the example of the agent negotiating the place of a meeting. Basic beliefs of the agent may include for instance the fact that "London is not warm", "Tunisia is hot", "London is very expensive", etc. This base may also contain some integrity constraints related to the dialog subject such as the meeting cannot be at the same time in London and in Tunisia.

We propose a formal framework for defining strategies [1]. This framework can be regarded as two separate systems: The first one takes as input the possible replies allowed by a protocol, a set of strategic beliefs and a set of strategic goals and returns the best speech act. The second system takes as input a set of alternatives, a set of basic beliefs and a set of functional goals and returns the best content of the chosen speech act. The two systems are grounded on argumentation theory. The basic idea behind each system is to construct the arguments in favor and against each choice, to compute the strength of each argument and finally to compare pairs of choices on the basis of the quality of their supporting arguments.

An extension of this work would be to study more deeply the links between the strategic and the functional goals of an agent. In this paper, the union of the two goals bases is supposed to be coherent. However, in reality it may be the case that an agent has a strategic goal which is incompatible with a functional one. Let us take the example of an agent negotiating the price of a car. This agent may have as a strategic goal to sell at the end of the dialog. It may have also the goal of selling his car with a high price. These two goals are not compatible since if the agent wants really to sell at the end its car, it should reduce the price.

\section{Acknowledgments}

This research is partially supported by the CG 64 Department (Conseil Général des Pyrénées Atlantiques), in the context of CAROSO Project.

\section{References}

[1] L. Amgoud and N. Hameurlain. An argumentation-based approach for dialog move selection. In Proceedings of the Third International Workshop on Argumentation in Multi-Agent Systems, (ArgMAS'06), LNCS/LNAI, 2006.

[2] D. N. Walton and E. C. W. Krabbe. Commitment in Dialogue: Basic Concepts of Interpersonal Reasoning. State University of New York Press, Albany, NY, 1995. 